\title{
MAPK/c-Jun signaling pathway contributes to the upregulation of the anti-apoptotic proteins Bcl-2 and Bcl-xL induced by Epstein-Barr virus-encoded $B A R F 1$ in gastric carcinoma cells
}

\author{
YUQIONG ZHANG ${ }^{1,2}$, MEIQIN XU ${ }^{3}$, XUEYI ZHANG ${ }^{4}$, FUYING CHU $^{1}$ and TIANJI ZHOU ${ }^{1}$ \\ ${ }^{1}$ Department of Clinical Laboratory and Microbiology, School of Medicine, Jiangsu University, Zhenjiang, Jiangsu 212013; \\ ${ }^{2}$ Department of Clinical Laboratory, Suzhou Municipal Hospital Affiliated to Nanjing Medical University, Suzhou, \\ Jiangsu 215168; ${ }^{3}$ Department of Clinical Laboratory, Suzhou Psychiatric Hospital, Suzhou, Jiangsu 215137; \\ ${ }^{4}$ Department of Biochemistry, School of Medicine, Jiangsu University, Zhenjiang, Jiangsu 212013, P.R. China
}

Received March 30, 2016; Accepted August 8, 2017

DOI: $10.3892 / \mathrm{ol} .2018 .8293$

\begin{abstract}
BARF1, encoded by Epstein-Barr virus (EBV), has been hypothesized to function as an oncogene, which was expressed in gastric carcinoma cells. Additionally, it has been reported that the anti-apoptotic function is closely associated with the expression of the B-cell lymphoma-2 (Bcl-2) protein. In addition, the signaling pathway has been reported to be involved in numerous diseases, including the mitogen-activated protein kinase (MAPK) cascade. In order to study the specific mechanism of anti-apoptotic function, $B A R F 1$-stably-expressing immortalized normal human embryo gastric epithelial cell line GES1 (GES-BARF1), and well-, moderately- and poorly-differentiated gastric carcinoma cell lines, MKN28 (which has been reported to be contaminated with the moderately-differentiated MKN74 gastric carcinoma cell line), SGC7901 and BGC823 (MKN-BARF1, $\mathrm{SGC}-B A R F 1$ and $\mathrm{BGC}-B A R F 1$, respectively) (GCC-BARF1) were constructed, with transfection of cells with the empty vector pSG5 acting as controls. Western blot analysis was performed to analyze the protein expression and the phosphorylation levels. Compared with the controls, it was found that the protein expression levels of c-Jun, Bcl-2 and B-cell lymphoma-extra large (Bcl-xL), as well as the phosphorylation levels of c-Jun, c-Jun N-terminal kinase (JNK) 1/2/3, p38 and extracellular signal-regulated kinase (ERK) $1 / 2$ proteins were upregulated in 3 GCC-BARF1 but not significantly changed in GES-BARF1. The expression levels of the c-Jun, Bcl-2 and $\mathrm{Bcl}-\mathrm{xL}$ proteins, and levels of c-Jun protein phosphorylation were significantly decreased in SGC-BARF1 cells subsequent
\end{abstract}

Correspondence to: Professor Tianji Zhou, Department of Clinical Laboratory and Microbiology, School of Medicine, Jiangsu University, 301 Xuefu Road, Zhenjiang, Jiangsu 212013, P.R. China E-mail: zhoutj64@ujs.edu.cn

Key words: Epstein-Barr virus, BARF1, JNK1/2/3-MAPK, p38-MAPK, ERK1/2-MAPK, gastric carcinoma, Bcl-2, Bcl-xL to treatment with SP600125, SB203580, and U0126, which were the specific inhibitors of JNK1/2/3, p38 and ERK1/2 respectively. In addition, there was a gradual increase in the protein expression and phosphorylation levels between normal gastric epithelial cells, and well-differentiated, moderately-differentiated and poorly-differentiated gastric carcinoma cells, but this was not statistically significant. Therefore, the present study hypothesized that JNK1/2/3-, p38- and ERK1/2-MAPK/c-Jun cascade signaling pathways may contribute to the upregulation of the expression of the anti-apoptotic proteins Bcl-2 and $\mathrm{Bcl}-\mathrm{xL}$ induced by BARF1 in gastric carcinoma cells. This mechanism may mainly work in the progressive phase of the development in EBV-associated gastric carcinoma.

\section{Introduction}

Epstein-Barr virus (EBV) belongs to the $\gamma$-herpes virus subfamily, which was identified and isolated from Burkitt's lymphoma cells. The virus is classified as an oncogenic virus associated with malignant tumors including nasopharyngeal carcinoma (NPC), gastric cancer (GC), Burkitt's lymphoma and Hodgkin's lymphoma $(1,2)$. Therefore, the presence of EBV in GC is occurs in $10 \%$ of case worldwide (3). The EBV-positive GC is termed as EBV-associated GC (EBVaGC) (4). EBV self-replicates in an episomal form extrachromosomally and expresses latent genes (5). According to the different host cell types, the appearance of viral latent genes are varied and categorized into three patterns, consisting of Latency I, II and III (6). EBVaGC belongs to the Latency I pattern, with latent gene products including EBV-encoded small RNAs (EBERs), EBV-determined nuclear antigen I (EBNAl) and BamHI-A rightward transcripts ( $B A R T s, B A R F O$ and $B A R F 1)$, not including $L M P 1$ and $E B N A 2$, which are usually expressed in NPC. The tumorigenic effect of $B A R F 1$ has been demonstrated in numerous types of cells, which are associated with stimulating cell proliferation and transformation $(7,8)$. In addition, $B A R F 1$ can be detected in almost all cases of EBVaGC, but not LMPI (7). Therefore, BARF1 is the only viral oncogene identified in EBVaGC at present and may have an important role in the development of EBVaGC. Nevertheless, the exact 
mechanism through which $B A R F 1$ induces $\mathrm{GC}$ remains elusive.

$B A R F 1$ has also been reported to have anti-apoptotic function. For instance, $\mathrm{N}$-terminal domain of $B A R F 1$ gene is able to activate anti-apoptotic protein B-cell lymphoma-2 (Bcl-2) expression in rodent fibroblasts (9). Bcl-2 activation has also been observed in EBV-negative human B lymphoma cell line Akata transfected by $B A R F 1$ (10). These findings indicate that $B A R F 1$ can resist apoptosis by activating $\mathrm{Bcl}-2$ expression. Transcriptional activator protein-1 (AP-1) is a heterodimer complex mainly composed of c-Fos and c-Jun, wherein c-Jun is the most important transcription factor. As an oncogenic protein, c-Jun has a key role in the regulation of cell proliferation, differentiation, invasion and apoptosis. Wang et al (3) analyzed and confirmed several transcription-associated genes including c-jun and c-fos were upregulated in the BARFI transfectants. The expression of the anti-apoptotic protein Bcl-2 was upregulated, while the expression of pro-apoptotic caspases and Bax protein was reduced.

In multicellular organisms, apoptosis is one of the most important forms of cell death, and usually presents disorders in human tumors (11). The abnormal activation or inhibition of signaling pathways is an important cause of apoptosis dysregulation, and the mitogen-activated protein kinase (MAPK) signaling pathway is one such pathway. When stimulated by various extracellular signals, c-Jun N-terminal kinase (JNK) is fully activated through phosphorylation of Thr183 and Tyr185, which can be mediated by the upstream MAPK kinase kinase 4 or MAPK kinase 7 and then has enzyme catalytic activity (12). Activation of JNK can enable the transcription factor c-Jun phosphorylation in ser63 and ser73, which improves the transcriptional activity. Additionally, activated c-Jun involved in the formation of AP-1 proteins and then to regulate a variety of proteins expression into the nucleus while formative AP-1 proteins are also capable of binding c-Jun promoter site itself to form positive feedback. Excessive activation of the extracellular regulated protein kinase $1 / 2$ (ERK1/2) signaling pathway has also been found in a variety of tumors, and is closely associated with the development of a variety of tumors. Regulation of apoptosis proteins is one of the main mechanisms that ERK1/2-MAPK act to resist apoptosis (13). In addition, another important member of MAPK, p38, which can also be activated in the cytoplasm or transfer to the nucleus to further regulate downstream substrates, such as the protein kinases Prak, MSK1/2 and transcription factor p53, ATF-2 and AP-1 to control cell proliferation, apoptosis and metastasis $(14,15)$.

Overall, the present study investigates whether BARF1 is capable of regulating $b c l-2$ expression through the JNK-, p38- and ERK-MAPK/c-Jun signaling pathway in gastric carcinoma cells.

\section{Materials and methods}

Cell culture and treatment. The immortalized normal human embryo gastric epithelial GES1 cell line and EBV-negative human gastric carcinoma MKN28, which has been reported to be contaminated with the moderately-differentiated MKN74 gastric carcinoma cell line (16), SGC7901 and BGC823 cell lines were purchased from the Chinese Academy of Sciences
Cell Bank (Shanghai, China). The pSG5 empty vector and pSG5-BARF1 stable transfectants, GES1-pSG5 (GES-SG), MKN28-pSG5 (MKN-SG), SGC7901-pSG5 (SGC-SG), BGC823-pSG5 (BGC-SG), and GES1-BARF1 (GES-BARF1), MKN28-BARF1 (MKN-BARF1), SGC7901-BARF1 (SGC-BARF1), BGC823-BARF1 (BGC-BARF1) (three cell clones of each), were constructed and preserved by the Key Laboratory of Laboratory Medicine of Jiangsu Province, School of Medicine (Jiangsu University, Zhenjiang, China). It was then identified by reverse transcription-quantitative polymerase chain reaction (RT-qPCR) that the expression of $B A R F 1$ was normal. Total RNA was extracted from cells using TRIzol (Invitrogen; Thermo Fisher Scientific, Inc., Waltham, MA, USA) and reverse transcription was performed using the Prime Script RT kit (Takara Bio, Inc., Otsu, Japan) according to the manufacturer's protocol. The levels of target mRNA in cells were analyzed by qPCR using SYBR Green I dye (Takara Bio, Inc.) detection. The PCR reactions were run at $95^{\circ} \mathrm{C}$ for $5 \mathrm{~min}$ followed by 30 cycles of $95^{\circ} \mathrm{C}$ for $30 \mathrm{sec}, 55^{\circ} \mathrm{C}$ for $30 \mathrm{sec}$ and finally $72^{\circ} \mathrm{C}$ for $5 \mathrm{~min}$.

Cells were maintained in Dulbecco's modified Eagle's medium (Invitrogen; Thermo Fisher Scientific, Inc.) growth medium plus $10 \%$ fetal bovine serum (Zhejiang Tianhang Biotechnology Co., Ltd., Zhejiang, China), $100 \mathrm{U} / \mathrm{ml}$ penicillin and $100 \mu \mathrm{g} / \mathrm{ml}$ streptomycin at $37^{\circ} \mathrm{C}$ in a humidified $5 \% \mathrm{CO}_{2}$ atmosphere. To study the effect of the JNK1/2/3, p38, and ERK1/2-MAPK/c-Jun signaling axes on the expression and phosphorylation of $\mathrm{Bcl}-2$ and $\mathrm{Bcl}-\mathrm{xL}$, the cells were separately treated with $25 \mu \mathrm{mol} / 1$ specific inhibitors of the above three signaling pathways, anthrapyrazolone (SP600125; targeted at the JNK1/2/3 pathway), 4-(4-fluorophenyl)-2-(4-methylsul finylphenyl)-5-(4-pyridyl)-1H-imidazole (SB203580; targeted at the p38 pathways) and 1,4-diamino-2,3-dicyano-1,4-bis (o-aminophenylmercapto) butadiene (U0126; targeted at the ERK1/2/MAPK/c-JUN pathway) (Beyotime Institute of Biotechnology, Haimen, China) at $37^{\circ} \mathrm{C}$ for $8 \mathrm{~h}$, and the same concentration of dimethyl sulfoxide (DMSO) (Sinopharm Chemical Reagent Co., Ltd., Shanghai, China) was used as a control.

Western blot analysis. Cells were cultured to a density of $\sim 75 \%$, and the inhibition tests were added with $50 \mathrm{~mol} / \mathrm{l}$ SP600125, SB203580, U0126 or DMSO to culture for $8 \mathrm{~h}$. Cells were lysed for total protein extraction in radioimmunoprecipitation assay buffer $(50 \mathrm{mM}$ Tris- $\mathrm{HCl}, \mathrm{pH} 7.4$; $150 \mathrm{mM} \mathrm{NaCl} ; 1 \%$ NP-40; $0.25 \%$ Na-deoxycholate; $1 \mathrm{mM}$ EDTA; $1 \mathrm{mM} \mathrm{NaF}$ ) together with a protease inhibitor cocktail (Sigma-Aldrich; Merck KGaA, Darmstadt, Germany). Cell lysate was collected, transferred into a 1.5-ml Eppendorf tube and clarified by centrifugation at $14,000 \mathrm{x}$ g for $5 \mathrm{~min}$ at $4^{\circ} \mathrm{C}$. The supernatant was collected and the protein concentration was determined using a BCA protein assay kit (Beyotime Institute of Biotechnology) according to the manufacturer's protocols.

Proteins (20 $\mu \mathrm{g} /$ lane) were separated by $10 \%$ SDS-PAGE and transferred to polyvinylidene difluoride membranes (Merck $\mathrm{KGaA}$ ). After blocking with $5 \%$ skim milk in $0.01 \mathrm{~mol} / 1 \mathrm{PBS}$ with Tween-20 (0.05\%) (PBST) for $1 \mathrm{~h}$ at room temperature, each membrane was incubated with the following primary antibodies in $5 \%$ skim milk overnight at $4^{\circ} \mathrm{C}$ in a humidified 
A

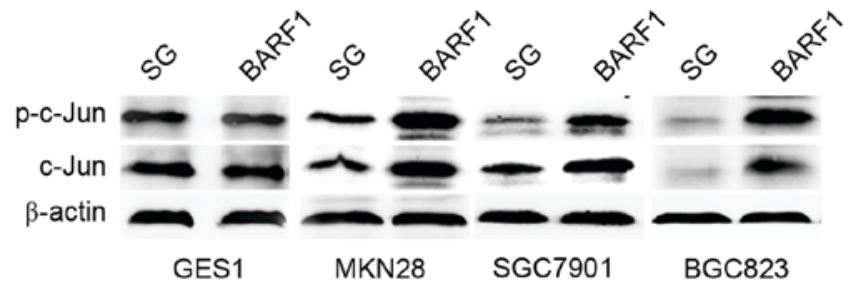

B

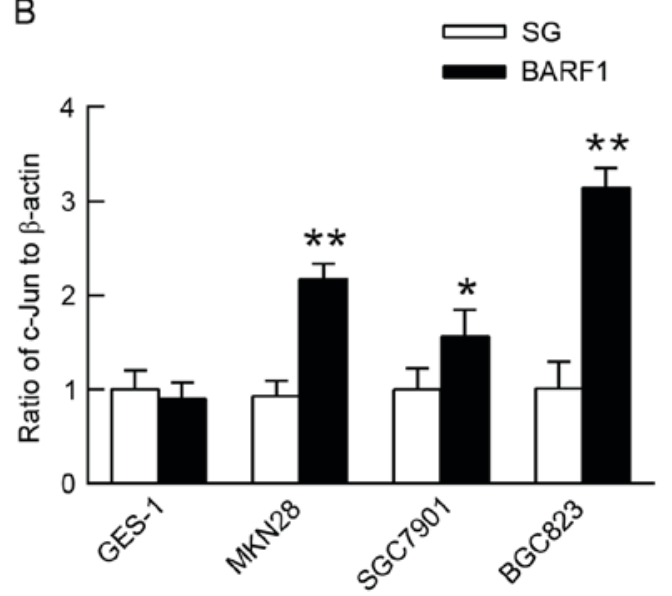

C

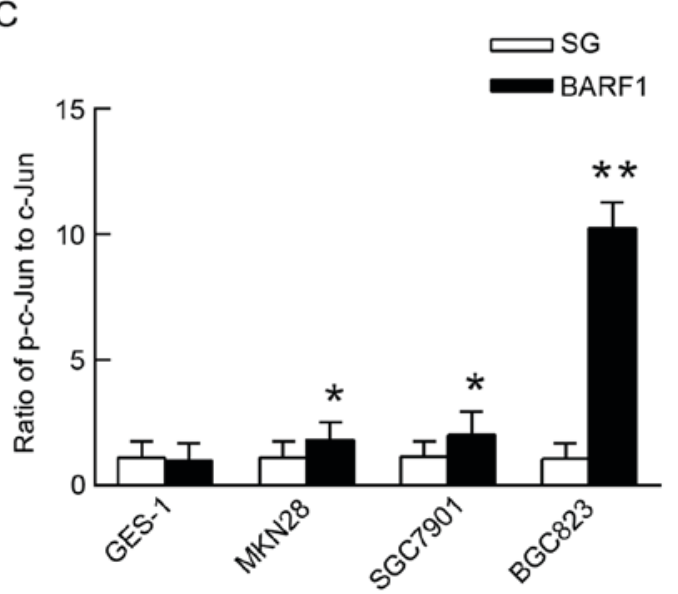

Figure 1. The expression and phosphorylation levels of the c-Jun protein in GEC-BARF1 cells. (A) Western blotting and quantitative analysis of (B) c-Jun protein expression and (C) phosphorylation in GEC-BARF1 cells compared with GEC-SG cells. Data were expressed as the mean \pm standard deviation from three independent experiments. ${ }^{*} \mathrm{P}<0.05,{ }^{* *} \mathrm{P}<0.01$ vs. GEC-SG.

chamber: Rabbit anti-c-Jun (9165T), anti-phosphorylated (p-) c-Jun (3270T), anti-ERK1/2 (4695T) and anti-p-ERK1/2 (4370T) (dilution, 1:1,000; Cell Signaling Technology, Inc., Danvers, MA, USA); anti-JNK1/2/3 (BS9939M), anti-p-JNK1/2/3 (BS4322), anti-p38 (BS9851M), anti-p-p38 (BS4635) (dilution, 1:1,000); anti-Bcl-2 (BS70205); and anti-Bcl-xL (BS1032) (dilution, 1:2,000; Bioworld Technology, Shanghai, China). The membranes were then incubated with horseradish peroxidase (HRP)-linked goat anti-rabbit IgG (BA1054; Wuhan Boster Biological Technology, Ltd., Wuhan, China) for $1 \mathrm{~h}$ at room temperature subsequent to washing with PBST. The membranes were stripped and re-probed with a mouse anti- $\beta$-actin antibody (BM0627; dilution, 1:3,000; Wuhan Boster Biological Technology, Ltd.) at $4^{\circ} \mathrm{C}$ overnight and then incubated with an HRP-linked goat anti-mouse immunoglobulin G antibody (BA1050; dilution, 1:3,000; Wuhan Boster Biological Technology, Ltd.) at room temperature for $1 \mathrm{~h}$. Immunoreactive proteins were detected by using an enhanced chemiluminescence system (GE Healthcare ImageQuant; GE Healthcare, Chicago, IL, USA). Relative protein expression levels were quantified by comparing the density of each band relative to the $\beta$-actin reference gene using Quantity One analysis software 4.62 (Bio-Rad Laboratories, Inc., Waltham, MA, USA). All experiments were independently performed three times at least.

Statistical analysis. Values were presented as the mean \pm standard deviation. Student's t-test was performed to evaluate differences between the GEC-BARF1 and GEC-SG groups. One-way analysis of variance was used to assess differences among the groups. Tukey's honest significant difference was used for post hoc analysis of the means. SPSS (version 19.0) software (IBM Corp., Armonk, NY, USA) was used for statistical analyses. Linear regression and linear correlation analysis were applied to trend analysis. $\mathrm{P}<0.05$ was considered to indicate a statistically significant difference.

\section{Results}

Expression and phosphorylation levels of the c-Jun protein are increased in GCC-BARF1 cells. In order to analyze the activity of c-Jun in GEC-BARF1, gastric epithelial cells were transfected with pSG5 empty vector (GEC-SG) as the control. The expression and phosphorylation levels of the c-Jun protein were then analyzed by western blot analysis in GEC-BARF1 cells. The results showed that the c-Jun protein expression and phosphorylation levels were significantly increased in GCC-BARF1 cells compared with GCC-SG cells $(\mathrm{MKN}-B A R F 1$ vs. MKN-SG, $\mathrm{P}<0.01$ and $\mathrm{P}<0.05$ for c-Jun expression and phosphorylation levels, respectively; SGC-BARF1 vs. SGC-SG, $\mathrm{P}<0.01$ and $\mathrm{P}<0.05$ for c-Jun expression and phosphorylation levels, respectively, BGC-BARF1 vs. BGC-SG, $\mathrm{P}<0.05$ and $\mathrm{P}<0.01$ for $\mathrm{c}-\mathrm{Jun}$ expression and phosphorylation levels, respectively), but not significantly changed in GES-BARF1 cells compared with GES-SG cells ( $\mathrm{P}>0.05)$. In addition, the c-Jun protein expression and phosphorylation levels in the GCC-BARF1 group were increased compared with the GES-BARF1 group (MKN-BARF1 vs. GES-BARF1, $\mathrm{P}<0.05$; SGC-BARF1 vs. GES-BARF1, $\mathrm{P}<0.05$; BGC-BARF1 vs. GES-BARF1, $\mathrm{P}<0.01$; Fig. 1).

JNK1/2/3, p38 and ERK1/2-MAPK/c-Jun cascade pathways are activated in GCC-BARF1 cells. To investigate whether the activation of c-Jun was associated with the JNK-MAPK 

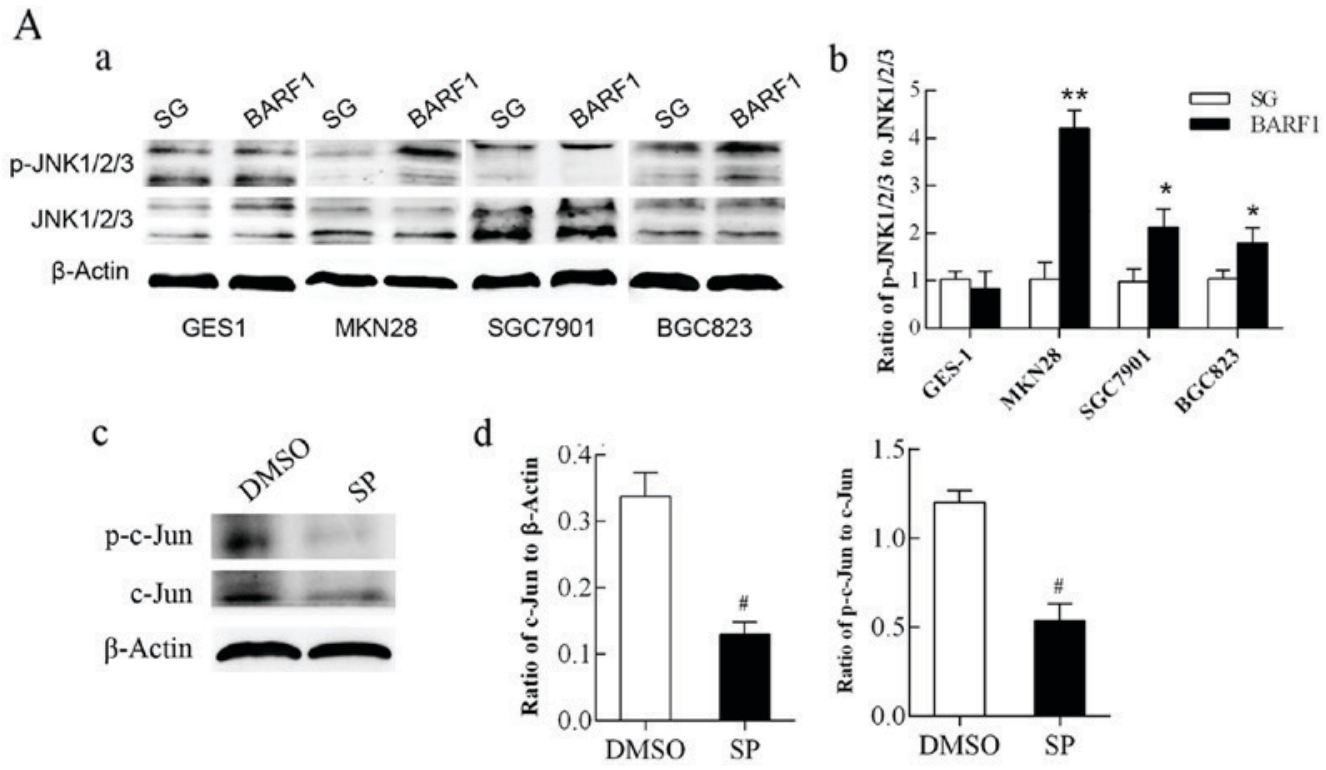

B a

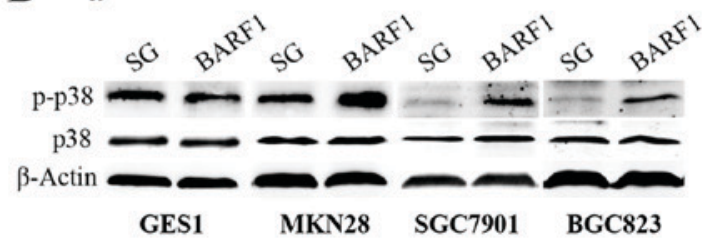

C
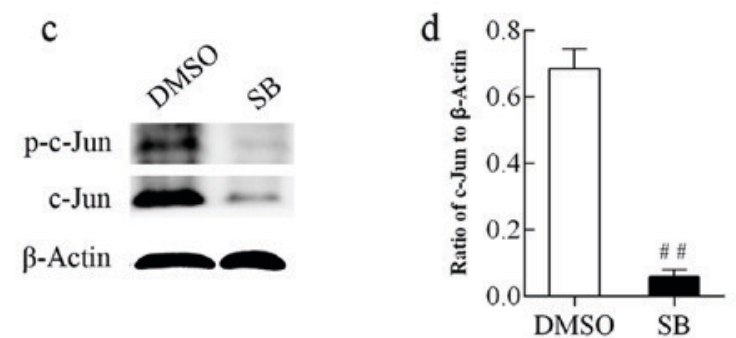

C a

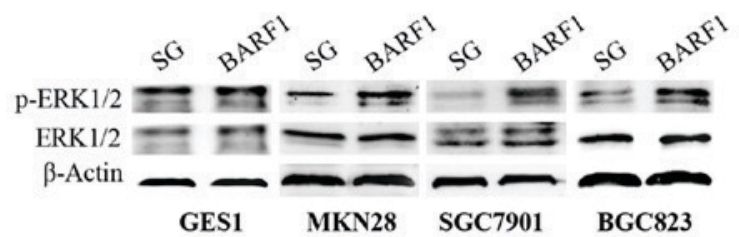

c

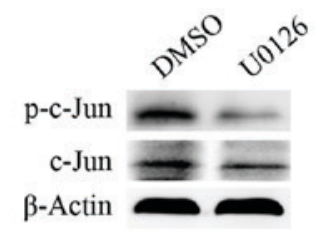

b
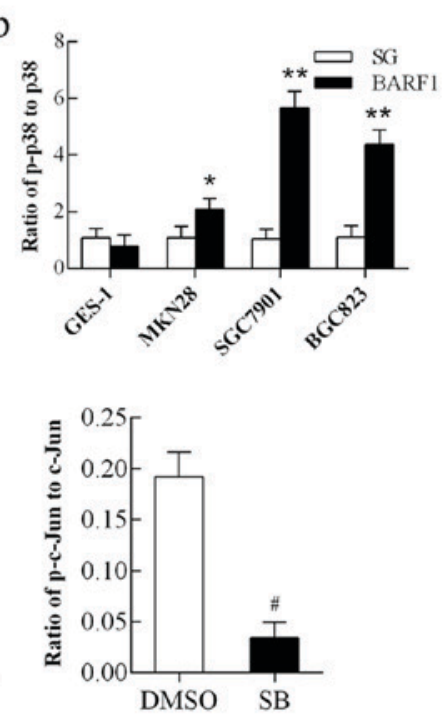

b
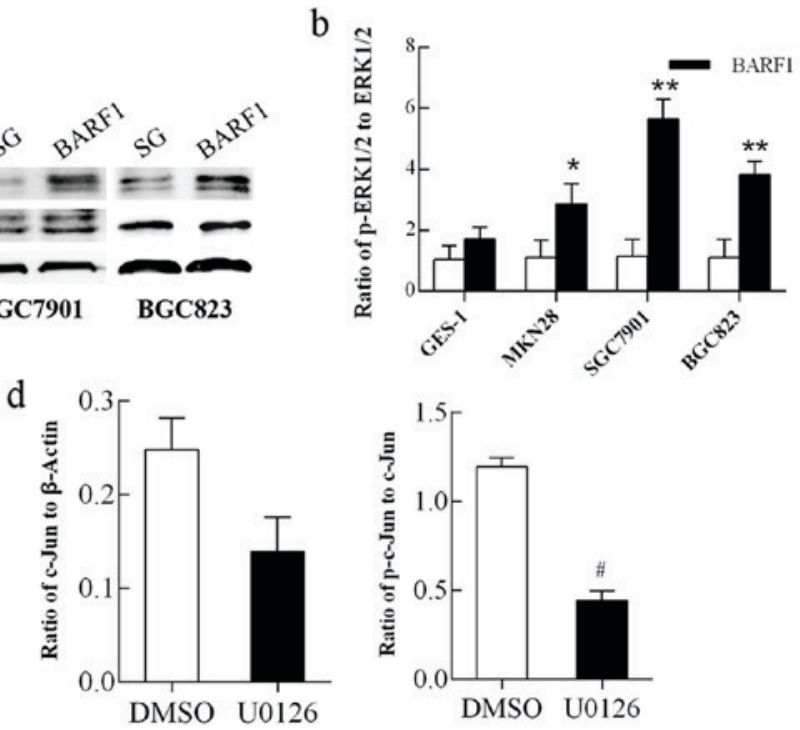

Figure 2. Analysis of the activity of JNK1/2/3, p38 and ERK1/2-MAPK/c-Jun signaling pathways in GEC-BARF1 cells. (A-a, B-a and C-a) Western blotting and (A-b, B-b and C-b) quantitative analysis of JNK1/2/3, p38 and ERK1/2 protein phosphorylation, respectively, in GEC cells. (A-c, B-c and C-c) Western blotting and (A-d, B-d and C-d) quantitative analysis of c-Jun expression and phosphorylation in SGC-BARF1 treated with SP600125, SB203580 and U0126 compared with DMSO, respectively. Data were expressed as the mean \pm standard deviation from three independent experiments. ${ }^{*} \mathrm{P}<0.05,{ }^{* *} \mathrm{P}<0.01$ vs. GEC-SG. ${ }^{*} \mathrm{P}<0.05$, ${ }^{\# \#} \mathrm{P}<0.01$ vs. DMSO control. DMSO, dimethyl sulfoxide. 
signaling pathway, western blot analysis was used to analyze the changes in the phosphorylation level of JNK1/2/3 proteins in the GEC-BARF1 group with GEC-SG as controls. The results showed that there was no significant difference in the phosphorylation level of $\mathrm{JNK} 1 / 2 / 3$ protein between GES-BARF1 cells and GES-SG cells (P>0.05), while the phosphorylation level of $\mathrm{JNK} 1 / 2 / 3$ proteins was significantly increased in GCC-BARF1 cells compared with GCC-SG cells (MKN-BARF1 vs. MKN-SG, P<0.01; SGC-BARF1 vs. SGC-SG, $\mathrm{P}<0.05$; $\mathrm{BGC}-B A R F 1$ vs. $\mathrm{BGC}-\mathrm{SG}, \mathrm{P}<0.05)$. Additionally, the phosphorylation levels of $\mathrm{JNK} 1 / 2 / 3$ protein in the GCC-BARF1 group was increased compared with that in the GES-BARF1 group (MKN-BARF1 vs. GES-BARF1, $\mathrm{P}<0.01$; SGC-BARF1 vs. GES-BARF1, $\mathrm{P}<0.01$; $\mathrm{BGC}-B A R F 1$ vs. GES-BARF1, $\mathrm{P}<0.05)$, and the phosphorylation level of JNK1/2/3 protein in MKN-BARF1 had the highest increase in the GCC-BARF1 group ( $\mathrm{P}<0.01$; Fig. $2 \mathrm{~A}-\mathrm{a}$ and $\mathrm{b})$. The expression and phosphorylation levels of c-Jun protein were significantly decreased in SGC-BARF1 cells subsequent to treatment with the specific inhibitor of JNK1/2/3 SP600125 $(\mathrm{P}<0.05$; Fig. 2A-c and d).

Western blotting showed that the phosphorylation level of the p38 protein was not significantly different $(\mathrm{P}>0.05)$ between GES-BARF1 and GES-SG groups, while there was a significant increase in GCC-BARF1 compared with GCC-SG (MKN-BARF1 vs. MKN-SG, P<0.05; SGC-BARF1 vs. SGC-SG, $\mathrm{P}<0.01$; BGC-BARF1 vs. BGC-SG, $\mathrm{P}<0.01$ ). The phosphorylation level of the p38 protein in the GCC-BARF1 group was significantly increased compared with the GES-BARF1 group (full $\mathrm{P}<0.01$ ), and the level in the SGC-BARF1 group was significantly increased compared with that in the MKN-BARF1 group $(\mathrm{P}<0.01)$ (Fig. 2B-a and $b$ ). Subsequent to treatment with the specific inhibitor of p38, SB203580, the expression and phosphorylation levels of c-Jun protein were significantly decreased $(\mathrm{P}<0.01$ and $\mathrm{P}<0.05$, respectively; Fig. 2B-c and d).

Western blot analysis showed that the phosphorylation level of ERK1/2 was not significantly different between GES-BARF1 and GES-SG groups ( $\mathrm{P}>0.05)$, while a significant increase occurred in the GCC-BARF1 group (MKN-BARF1 vs. MKN-SG, $\mathrm{P}<0.05$; SGC-BARF1 vs. SGC-SG, $\mathrm{P}<0.01$; BGC-BARF1 vs. BGC-SG, $\mathrm{P}<0.01)$. Additionally, the phosphorylation level of ERK1/2 in the GCC-BARF1 group was increased compared with that in the GES-BARF1 group except MKN-BARF1 (MKN-BARF1 vs. GES-BARF1, P>0.05; SGC-BARF1 vs. GES-BARF1, $\mathrm{P}<0.01$; BGC-BARF1 vs. GES-BARF1, $\mathrm{P}<0.05)$, and SGC-BARF1 cells had the highest phosphorylation level in the GEC-BARF1 group $(\mathrm{P}<0.05$; Fig. 2C-a and b). Subsequent to treatment with the specific inhibitor of ERK1/2, U0126, the phosphorylation level of the c-Jun protein in SGC-BARFl was significantly decreased $(\mathrm{P}<0.05)$, while the expression of $\mathrm{c}$-Jun was not evidently decreased ( $\mathrm{P}>0.05$; Fig. 2C-c and d).

JNK1/2/3, p38 and ERK1/2-MAPK/c-Jun signaling pathways mediated upregulation of the anti-apoptotic proteins Bcl-2 and Bcl-xL. To further investigate whether the role of these pathways mediated $B A R F 1$-induced apoptosis inhibition in GC cells, GEC-SG was used as a control and the expression of anti-apoptotic proteins $\mathrm{Bcl}-2$ and $\mathrm{Bcl}-\mathrm{xL}$ was analyzed in
GEC-BARF1 using immunoblotting. The results showed that there were no significant difference between GES-BARF1 and GES-SG $(\mathrm{P}>0.05)$, but the expression levels of $\mathrm{Bcl}-2$ and $\mathrm{Bcl}-\mathrm{xL}$ proteins in the GCC-BARF1 group were significantly increased compared with the GCC-SG group (MKN-BARF1 vs. $\mathrm{MKN}-\mathrm{SG}, \mathrm{P}<0.05$; $\mathrm{SGC}-B A R F 1$ vs. $\mathrm{SGC}-\mathrm{SG}, \mathrm{P}<0.01$; BGC-BARF1 vs. BGC-SG, $\mathrm{P}<0.01)$, and the GES-BARF1 groups (MKN-BARF1 vs. GES-BARF1; SGC-BARF1 vs. GES-BARF1, BGC-BARF1 vs. GES-BARF1; $\mathrm{P}<0.05, \mathrm{P}<0.01$, $\mathrm{P}<0.01$, and all $\mathrm{P}<0.01$; Fig. $3 \mathrm{~A}$ ).

To further understand the association between the anti-apoptotic proteins Bcl-2 and Bcl-xL, and the JNK1/2/3, p38 and ERK1/2-MAPK/c-Jun signaling pathways, western blotting was performed to analyze the protein expression levels of Bcl-2 and Bcl-xL in SGC-BARF1 subsequent to treatment with SP600125, SB203580 and U0126, respectively. The results then showed that the protein expression levels of Bcl-2 and Bcl-xL were significantly decreased subsequent to treatment with 3 inhibitors (SGC-BARF1-SP vs. SGC-BARF1-DMSO $\mathrm{P}<0.05$ and $\mathrm{P}<0.01$ for $\mathrm{Bcl}-2$ and $\mathrm{Bcl}-\mathrm{xL}$, respectively; SGC-BARF1-SB vs. SGC-DMSO, $\mathrm{P}<0.01$ for Bcl-2 and Bcl-xL; SGC-BARF1-U vs. SGC-BARF1-DMSO, $\mathrm{P}<0.05$ for Bcl-2 and Bcl-xL; Fig. 3B-D).

\section{Discussion}

To the best of our knowledge, the present study revealed for the first time that JNK1/2/3, p38 and ERK1/2-MAPK/c-Jun cascade signaling pathways participate to mediate the upregulation of anti-apoptotic protein Bcl-2 and Bcl-xL induced by EBV-encoded BARF1 in 3 gastric carcinoma cell lines in comparison with the normal gastric epithelium GES1 cells. It has been confirmed in a number of studies that BARF1 can inhibit cell apoptosis by activating anti-apoptotic protein Bcl-2 expression $(3,9,10)$. However, the precise mechanism by which $B A R F 1$ regulates Bcl-2 expression remains unclear. In order to analyze the mechanism, the immortalized normal human embryo gastric epithelial GES1 cell line, and three gastric carcinoma cell lines in different differentiation stages, consisting of well, moderately and poorly-differentiated cells (MKN28, SGC7901 and BGC823, respectively), were used in the present study.

AP-1 is a complex composed of homologous or heterologous dimers of Jun and Fos family members. The AP-1 protein activates target gene transcription activity by binding to its DNA, and then contributes to a series of pathophysiological processes, including cell proliferation, apoptosis and differentiation (17). c-Jun is the fundamental member of the AP-1 family, and is also the most powerful transcriptional activator. As an oncoprotein, the c-Jun/AP-1 protein shows overexpression in breast cancer, ovarian cancer, endometrial cancer, colon cancer and lung cancer (17-19), and can also promote skin cancer and liver cancer development (20,21). For example, in terms of cell apoptosis, Eferl et al (22) showed that c-Jun-deficient mouse embryos can promote the apoptosis of fetal rat hepatocytes. Additionally, Eferl et al (20) demonstrated that c-Jun/AP-1 resisted TNF- $\alpha$-induced liver cell apoptosis by inhibiting the expression of the apoptotic protein p53. In the present study, it was found that BARF1 promotes the phosphorylation of the c-Jun protein in gastric carcinoma cells. The phosphorylation of c-Jun first triggers activation of 


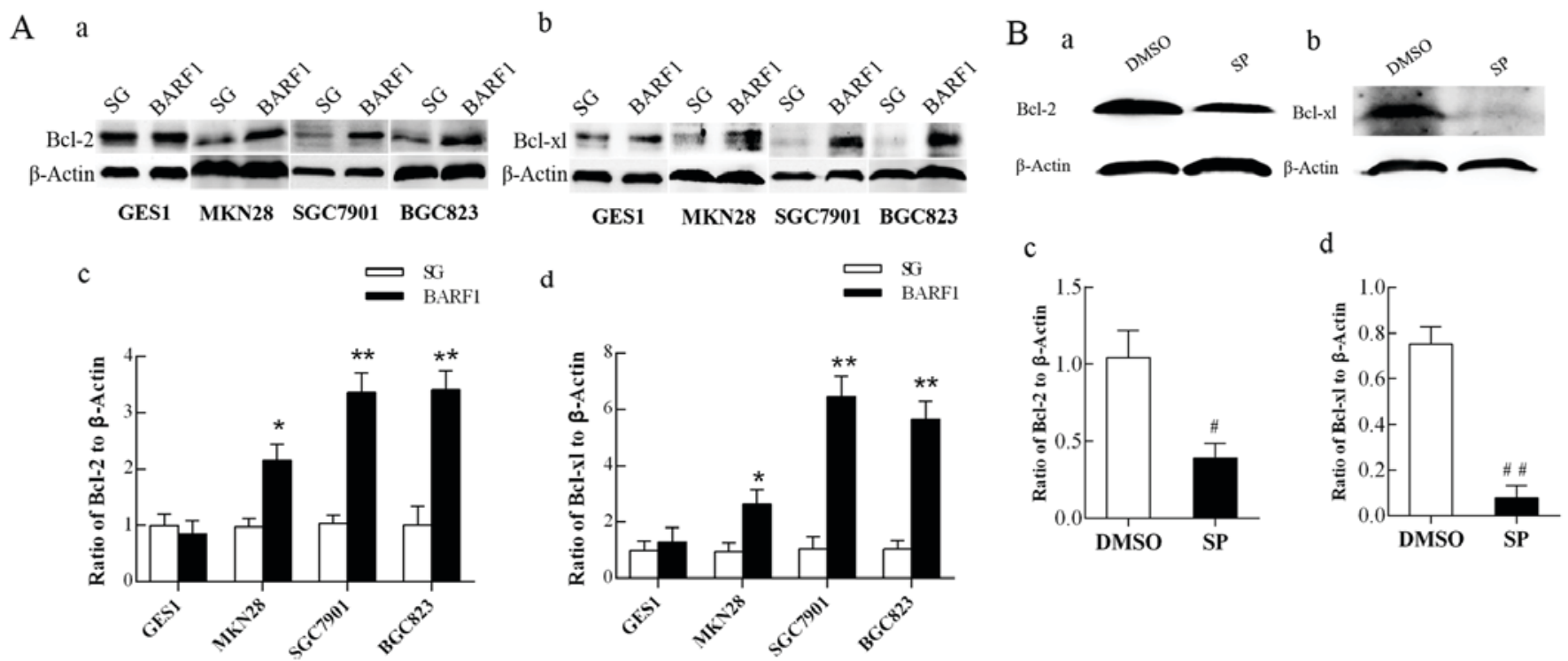

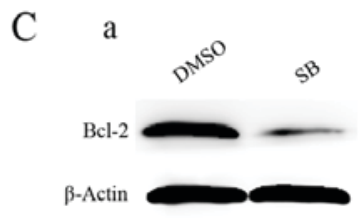
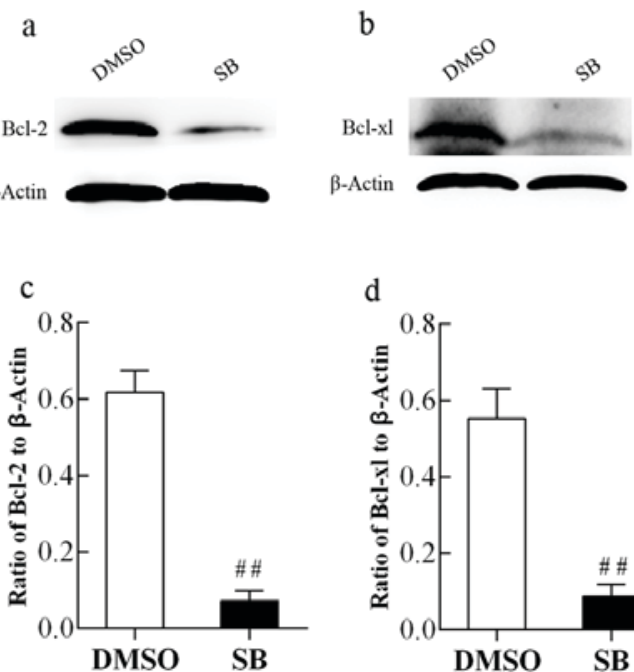

$\mathrm{d}$

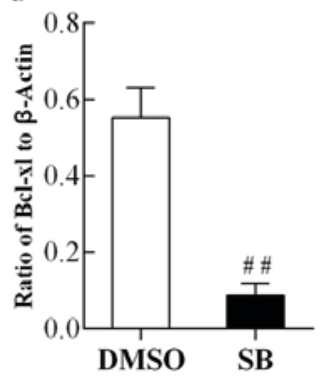

D a

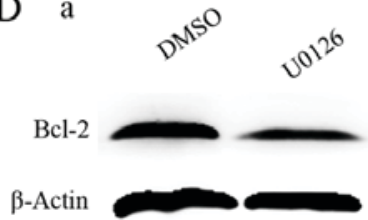

c

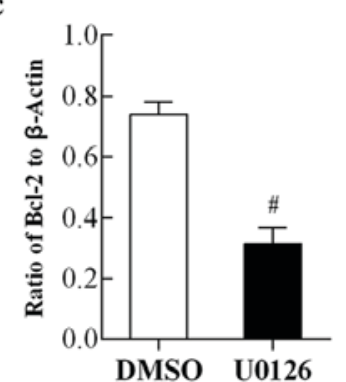

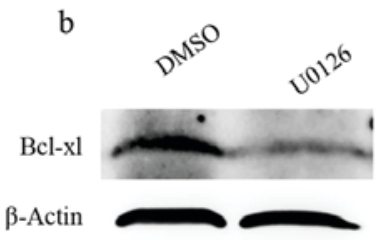

d

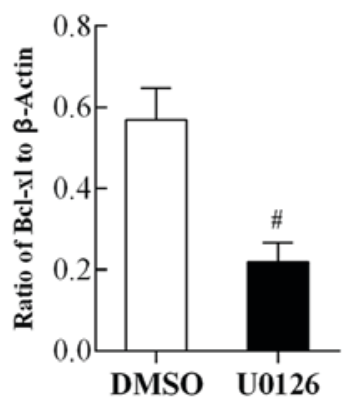

Figure 3. Analysis of Bcl-2 and Bcl-xL protein expression in GEC-BARF1 cells. Western blotting of (A-a) Bcl-2 and (A-b) Bcl-xL expression, and quantitative analysis of (A-c) Bcl-2 and (A-d) Bcl-xL expression in GEC-BARF1 cells compared with GEC-SG cells. (B-a, B-b, C-a, C-b, D-a and D-b) Western blotting and quantitative analysis of (B-c, C-c and D-c) Bcl-2 and (B-d, C-d and D-d) Bcl-xL protein expression levels in SGC-BARF1 treated with SP600125, SB203580 and U0126 compared with DMSO, respectively. Data were expressed as the mean \pm standard deviation from three independent experiments. ${ }^{*} \mathrm{P}<0.05,{ }^{* *} \mathrm{P}<0.01$ vs. GEC-SG. " $\mathrm{P}<0.05,{ }^{\# \#} \mathrm{P}<0.01$ vs. DMSO control. DMSO, dimethyl sulfoxide; Bcl, B-cell lymphoma.

AP-1. In turn, activated c-Jun/AP-1 interacts with the c-Jun promoter to promote the synthesis of c-Jun/AP-1 to form the positive feedback regulation of the Jun gene, which is important in signal transduction into long-term effects on cellular gene expression (16). Previously, it has been found that several transcription associated genes, including $c$-jun and c-fos, were upregulated in the BARF1 transfectant (BGC823-BARF1) (3). Similar to the aforementioned research, the present study found that the increase of c-Jun at the protein level by western blotting. As a transcription factor, c-Jun may be adjusted both at the protein expression and the phosphorylation levels, and improvement of any level is able to promote its downstream target gene expression.

Activity of c-Jun is regulated by a variety of upstream factors. Among these factors, JNK is a member of the MAPK family, and is a highly-conserved serine/threonine protein kinase. In mammals, JNK and p38-MAPK are described as stress activated protein kinases (SAPKs) that promote cell death $(23,24)$ and mediate inflammatory response $(25,26)$. The JNK protein kinase is encoded by 3 homologous genes, consisting of JNK1, JNK2 and JNK3. The JNK/SAPK signaling pathway has two roles in the regulation of cell apoptosis (27). For example, activation of the JNK/c-Jun signaling pathway can promote apoptosis of neural cells. However, occasionally, c-Jun does not participate in the JNK-mediated apoptosis effect. Hilberg et al (28) showed that in Jnk $1^{-1-}$ $\mathrm{Jnk}^{2-1-}$ mice, the neuronal cells exhibited impaired apoptosis, while nerve cells did not appear to have impaired apoptosis in c-Jun-/-mice. This indicates that JNK1 and JNK2 induced the apoptosis of neuronal cells is not always mediated by c-Jun, another factor may be involved in this process. Subsequently, Raitano et al (29) found that the $b c r$-abl leukemia oncogene activates the JNK/SAPK pathway of hematopoietic stem cells and then increase the capacity of cell transformation by activating downstream c-Jun protein. When c-Jun or JNK is repressed, the transformation function of $b c r-a b l$ is also 
inhibited. Hess et al (30) showed that $b c r$-abl can further up-regulate $b c l-2$ expression by activating the JNK/SAPK pathway and result in enhanced transformation ability of $\mathrm{B}$ lymphoblastoid cells accompany with diminished apoptosis. Consequently, it can be speculated that $b c r$ - $a b l$-induced activation of JNK/c-Jun may promote $b c l-2$ expression and mediate cell transformation ability, accompanied by anti-apoptosis function.

In the present study, the phosphorylation level of JNK $1 / 2 / 3$, and c-Jun expression and phosphorylation levels were found to be increased in the GCC-BARF1 group, and significantly decreased in the SGC-BARF1 group subsequent to treatment with the specific inhibitor of JNK1/2/3, suggesting that BARF1 promoted c-Jun activity at both protein expression and phosphorylation levels by activating JNK1/2/3-MAPK signaling, and consequentially regulated the expression of downstream target genes.

As a regulator of cell death, p38-MAPK plays a dual role in mediating cell death or cell survival, and the function of p38-MAPK is determined by the type of stimulus or a specific cell type. Activated p38 can act on a variety of transcription factors, such as STAT1, NF- $\kappa$ B and AP-1, and then regulates cell proliferation, apoptosis, metastasis and differentiation. p38-MAPK can enhance the anti-apoptosis ability of cells that are mostly associated with drug resistance. Previously, Milone et al (31) described that the activation of the p38-MAPK signaling pathway in prostate cancer cells leads to resistance of zoledronic acid-induced apoptosis and overexpression of the anti-apoptotic protein $\mathrm{Bcl}-2$. It was also found that the p38-MAPK signaling pathway augments the expressions of associated factors, such as MMP-2, VEGF and IL-2, and cause epithelial-to-mesenchymal transition. It shows that p38-MAPK signal pathway may promote drug resistance in numerous aspects, such as transfer, proliferation and anti-apoptosis. Consistent with the aforementioned studies, in the present study, it was found that when the p38-MAPK signaling pathway is specifically suppressed by SB203580, Bcl-2 and Bcl-xL protein expression, as well as the expression and phosphorylation levels of c-Jun, was significantly decreased, indicating that $B A R F 1$ performs its anti-apoptotic effect by activating the p38-MAPK/c-Jun pathway in gastric carcinoma cells.

Zelivianski et al (32) found that the activated ERK pathway mediates an anti-apoptotic response to the treatment with certain chemotherapeutic agents. Nishinaka et al (33) found that phorbol ester, 12-O-tetradecanoyl phorbol 13-acetate can induce c-Jun expression in an ERK-dependent manner in human lung cancer cells. A previous study has indicated that BARF1 may upregulate $\mathrm{Bcl}-2$ expression through the ERK1/2-MAPK/c-Jun signaling pathway in NPC cells. In the present study, it was observed that ERK1/2 was activated by $B A R F 1$, and c-Jun protein expression and phosphorylation levels were significantly increased in GCC-BARF1 cells After SGC-BARF1 cells were treated with U0126, the phosphorylation level of the c-Jun protein was markedly decreased, although there was no significant alteration in protein expression, indicating that ERK1/2 regulated c-Jun activation mainly at the phosphorylation level. Accordingly, this indicated that the ERK signaling pathway may be involved in BARF1-induced apoptosis inhibition in gastric carcinoma cells.
c-Jun/AP-1 regulates cell apoptosis by a variety of mechanisms (34). Li et al (35) has shown that the c-Jun protein can enhance the transcription level of $b c l-2$ by binding to the $b c l-2$ promoter in human endometrial glandular cells. In agreement with a previous study (3), it was observed that the expression of Bcl-2 was significantly increased in GCC-BARF1 compared with GCC-SG, and there was a gradual increase in expression that was associated with the malignant degree of cells and showed a certain association with c-Jun activity, but this was not statistically significant. Following treatment with three specific inhibitors of the MAPK signaling pathway, c-Jun protein and phosphorylation levels, as well as Bcl-2 and Bcl-xL protein expression levels, were significantly decreased, indicating JNK1/2/3, p38 and ERK1/2-MAPK/c-Jun signaling pathway were involved in the regulation of the $\mathrm{Bcl}-2$ and $\mathrm{Bcl}-\mathrm{xL}$ proteins in gastric carcinoma cells.

Furthermore, the phosphorylation levels of p38 and ERK1/2 proteins in SGC-BARF1 and of JNK1/2/3 proteins in $\mathrm{MKN}-B A R F 1$ was significantly increased compared with that in the other two cells in the GCC-BARF1 group, while the expression levels of c-Jun, Bcl-2 and Bcl-xL and phosphorylation levels of c-Jun, JNK1/2/3, p38 and ERK1/2 were not significantly different in GES-BARF1 cells compared with GES-SG cells, suggesting that the p38 and ERK1/2-MAPK/ c-Jun signaling pathways may be dominant in the progressive stage, and JNK1/2/3-MAPK/c-Jun may mainly occur in the early stage of EBVaGC development. However, the MAPK/ c-Jun signaling cascade is unlikely to play an important role in the initial stage of EBVaGC.

Previously, it been reported that the MKN28 cell line may be cross-contaminated with MKN74 cells which are moderately-differentiated gastric carcinoma cell lines (16). Even if MKN74 cross-contaminated MKN28 cell line has been used in the present study, it is unlikely to affect the conclusions derived from the comparisons between BARF1 and SG-transfected cells and between the GCC and GES1 groups. However, the reliability of the conclusions about the functions of the 3 MAPK pathways in the different phases of EBVaGC evolution may have been affected, by having been derived from the comparison among 3 differently differentiated gastric carcinoma cell lines.

Chen et al (36) constructed a transfectant of B-cell-specific Moloney murine leukemia virus integration site 1 (Bmi-1) stably expressed (GES-Bmi-1) and find that overexpression of Bmi-1 can promote cell proliferation and inhibit apoptosis, and also enhance cell migration and invasion ability by upregulating vimentin and fibronectin via the PI3K/Akt signaling pathway. Accordingly, whether PI3K/Akt signaling pathway is involved in $B A R F 1$ mediated regulation on apoptosis of GES is worthy of further study. Furthermore, numerous issues required further exploration. Signaling networks are complicated, and whether other signaling pathways are involved in the activation of c-Jun to participate in the anti-apoptotic effect of $B A R F 1$ in gastric carcinoma requires investigation. Apart from the anti-apoptotic effect, whether c-Jun is also involved in other biological behavior, including cell proliferation, migration and invasion also requires additional investigation. These issues will be further explored in a following study. 


\section{Acknowledgements}

The present study was supported by the Professional Research Foundation for Advanced Talents of Jiangsu University (grant no. 06JDG011) to Tianji Zhou and Student's Scientific Research of Jiangsu University to Yuqiong Zhang.

\section{References}

1. Seto E, Yang L, Middeldorp J, Sheen TS, Chen JY, Fukayama M, Eizuru Y, Ooka T and Takada K: Epstein-Barr virus (EBV)-encoded BARF1 gene is expressed in nasopharyngeal carcinoma and EBV-associated gastric carcinoma tissues in the absence of lytic gene expression. J Med Virol 76: 82-88, 2005.

2. Zhang Y, Ohyashiki JH, Takaku T, Shimizu N and Ohyashiki K: Transcriptional profiling of Epstein-Barr virus (EBV) genes and host cellular genes in nasal NK/T-cell lymphoma and chronic active EBV infection. Br J Cancer 94: 599-608, 2006.

3. Wang Q, Tsao SW, Ooka T, Nicholls JM, Cheung HW, Fu S, Wong YC and Wang X: Anti-apoptotic role of BARF1 in gastric cancer cells. Cancer Lett 238: 90-103, 2006.

4. Takada K: Epstein-Barr virus and gastric carcinoma. Mol Pathol 53: 255-261, 2000.

5. Klein E: The complexity of the Epstein-Barr virus infection in humans. Pathol Oncol Res 4: 3-7, 1998.

6. Rowe M, Lear AL, Croom-Carter D, Davies AH and Rickinson AB: Three pathways of Epstein-Barr virus gene activation from EBNA1-positive latency in B lymphocytes. J Virol 66: 122-131, 1992.

7. zur Hausen A, Brink AA, Craanen ME, Middeldorp JM, Meijer CJ and van den Brule AJ: Unique transcription pattern of Epstein-Barr virus (EBV) in EBV-carrying gastric adenocarcinomas: Expression of the transforming BARF1 gene. Cancer Res 60: 2745-2748, 2000.

8. Wei MX, de Turenne-Tessier M, Decaussin G, Benet G and Ooka T: Establishment of a monkey kidney epithelial cell line with the BARF1 open reading frame from Epstein-Barr virus. Oncogene 14: 3073-3081, 1997.

9. Sheng W, Decaussin G, Sumner S and Ooka T: N-terminal domain of BARF1 gene encoded by Epstein-Barr virus is essential for malignant transformation of rodent fibroblasts and activation of BCL-2. Oncogene 20: 1176-1185, 2001.

10. Sheng W, Decaussin G, Ligout A, Takada K and Ooka T: Malignant transformation of Epstein-Barr virus-negative Akata cells by introduction of the BARF1 gene carried by Epstein-Barr virus. J Virol 77: 3859-3865, 2003.

11. Bai L and Wang S: Targeting apoptosis pathways for new cancer therapeutics. Annu Rev Med 65: 139-155, 2014.

12. Plotnikov A, Zehorai E, Procaccia S and Seger R: The MAPK cascades: Signaling components, nuclear roles and mechanisms of nuclear translocation. Biochim Biophys Acta 1813: 1619-1633, 2011.

13. Balmanno K and Cook SJ: Tumour cell survival signalling by the ERK1/2 pathway. Cell Death Differ 16: 368-377, 2009.

14. Ohashi M, Fogg MH, Orlova N, Quink C and Wang F: An Epstein-Barr virus encoded inhibitor of colony stimulating factor-1 signaling is an important determinant for acute and persistent EBV infection. PLoS Pathog 8: e1003095, 2012.

15. Iyoda K, Sasaki Y, Horimoto M, Toyama T, Yakushijin T, Sakakibara M, Takehara T, Fujimoto J, Hori M, Wands JR and Hayashi N: Involvement of the p38 mitogen-activated protein kinase cascade in hepatocellular carcinoma. Cancer 97: 3017-3026, 2003.

16. Angel P, Hattori K, Smeal T and Karin M: The jun proto-oncogene is positively autoregulated by its product, Jun/AP-1. Cell 55: 875-885, 1988.

17. Meng Q and Xia Y: c-Jun, at the crossroad of the signaling network. Protein Cell 2: 889-898, 2011.
18. Neyns B, Katesuwanasing, Vermeij J, Bourgain C, Vandamme B, Amfo K, Lissens W, DeSutter P, Hooghe-Peters E and DeGrève J: Expression of the jun family of genes in human ovarian cancer and normal ovarian surface epithelium. Oncogene 12: 1247-1257, 1996.

19. Vleugel MM, Greijer AE, Bos R, van der Wall E and van Diest PJ: c-Jun activation is associated with proliferation and angiogenesis in invasive breast cancer. Hum Pathol 37: 668-674, 2006.

20. Eferl R, Ricci R, Kenner L, Zenz R, David JP, Rath M and Wagner EF: Liver tumor development. c-Jun antagonizes the proapoptotic activity of p53. Cell 112: 181-192, 2003.

21. Young MR, Li JJ, Rincón M, Flavell RA, Sathyanarayana BK, Hunziker R and Colburn N: Transgenic mice demonstrate AP-1 (activator protein-1) transactivation is required for tumor promotion. Proc Natl Acad Sci USA 96: 9827-9832, 1999.

22. Eferl R, Sibilia M, Hilberg F, Fuchsbichler A, Kufferath I, Guertl B, Zenz R, Wagner EF and Zatloukal K: Functions of c-Jun in liver and heart development. J Cell Biol 145: 1049-1061, 1999.

23. Harper SJ and LoGrasso P: Signalling for survival and death in neurones: The role of stress-activated kinases, JNK and p38. Cell Signal 13: 299-310, 2001

24. Rawal N, Parish C, Castelo-Branco G and Arenas E: Inhibition of JNK increases survival of transplanted dopamine neurons in Parkinsonian rats. Cell Death Differ 14: 381-383, 2007.

25. Adhikary G, Sun Y and Pearlman E: C-Jun NH2 terminal kinase (JNK) is an essential mediator of Toll-like receptor 2-induced corneal inflammation. J Leukoc Biol 83: 991-997, 2008.

26. Ruano D, Revilla E, Gavilán MP, Vizuete ML, Pintado C, Vitorica $J$ and Castaño A: Role of p38 and inducible nitric oxide synthase in the in vivo dopaminergic cells' degeneration induced by inflammatory processes after lipopolysaccharide injection. Neuroscience 140: 1157-1168, 2006.

27. Lin A: Activation of the JNK signaling pathway: Breaking the brake on apoptosis. Bioessays 25: 17-24, 2003.

28. Hilberg F, Aguzzi A, Howells N and Wagner EF: c-jun is essential for normal mouse development and hepatogenesis. Nature 365: 179-181, 1993.

29. Raitano AB, Halpern JR, Hambuch TM and Sawyers CL: The Bcr-Abl leukemia oncogene activates Jun kinase and requires Jun for transformation. Proc Natl Acad Sci USA 92: 11746-11750, 1995.

30. Hess P, Pihan G, Sawyers CL, Flavell RA and Davis RJ: Survival signaling mediated by c-Jun NH (2)-terminal kinase in transformed B lymphoblasts. Nat Genet 32: 201-205, 2002.

31. Milone MR, Pucci B, Bruzzese F, Carbone C, Piro G, Costantini S, Capone F, Leone A, Di Gennaro E, Caraglia M and Budillon A: Acquired resistance to zoledronic acid and the parallel acquisition of an aggressive phenotype are mediated by p38-MAP kinase activation in prostate cancer cells. Cell Death Dis 4: e641,2013.

32. Zelivianski S, Spellman M, Kellerman M, Kakitelashvilli V, Zhou XW, Lugo E, Lee MS, Taylor R, Davis TL, Hauke R and Lin MF: ERK inhibitor PD98059 enhances docetaxel-induced apoptosis of androgen-independent human prostate cancer cells. Int J Cancer 107: 478-485, 2003.

33. Nishinaka T, Miura T, Sakou M, Hidaka C, Sasaoka C, Okamura A, Okamoto A and Terada T: Down-regulation of aldo-keto reductase AKR1B10 gene expression by a phorbol ester via the ERK/c-Jun signaling pathway. Chem Biol Interact 234: 274-281, 2015.

34. Shaulian E and Karin M: AP-1 as a regulator of cell life and death. Nat Cell Biol 4: E131-E136, 2002.

35. Li ZL, Abe H, Ueki K, Kumagai K, Araki R and Otsuki Y: Identification of c-Jun as bcl-2 transcription factor in human uterine endometrium. J Histochem Cytochem 51: 1601-1609, 2003.

36. Chen Y, Lian G, Zhang Q, Zeng L, Qian C, Chen S and Huang K: Overexpression of Bmi-1 induces the malignant transformation of gastric epithelial cells in vitro. Oncol Res 21: 33-41, 2013. 